\title{
Theory of Spinors in Curved Space-Time
}

\author{
Ying-Qiu Gu* \\ School of Mathematical Science, Fudan University, Shanghai 200433, China
}

The interaction between spinors and gravity is the most complicated and subtle interaction in the universe, which involves the basic problem to unified quantum theory and general relativity. By means of Clifford Algebra, a unified language and tool to describe the rules of nature, this paper systematically discusses the dynamics and properties of spinor fields in curved space-time, such as the decomposition of the spinor connection, the classical approximation of Dirac equation, the energy momentum tensor of spinors and so on. To split spinor connection into Keller connection $\Upsilon_{\mu} \in \Lambda^{1}$ and pseudo-vector potential $\Omega_{\mu} \in \Lambda^{3}$ by Clifford algebra not only makes the calculation simpler, but also highlights their different physical meanings. The representation of the new spinor connection is dependent only on the metric, but not on the Dirac matrix. Keller connection only corresponds to geometric calculations, but the potential $\Omega_{\mu}$ has dynamical effects, which couples with the spin of a spinor and may be the origin of the celestial magnetic field. Only in the new form of connection can we clearly define the classical concepts for the spinor field and then derive its complete classical dynamics, that is, Newton's second law of particles. To study the interaction between space-time and fermion, we need an explicit form of the energy-momentum tensor of spinor fields. However, the energy-momentum tensor is closely related to the tetrad, and the tetrad cannot be uniquely determined by the metric. This uncertainty increases the difficulty of deriving rigorous expression. In this paper, through a specific representation of tetrad, we derive the concrete energy-momentum tensor and its classical approximation. In the derivation of energy-momentum tensor, we obtain a spinor coefficient table $S_{a b}^{\mu \nu}$, which plays an important role in the interaction between spinor and gravity. From this paper we find that, Clifford algebra has irreplaceable advantages in the study of geometry and physics.

Keywords: Clifford algebra; tetrad; spinor connection; natural coordinate system; energymomentum tensor; local Lorentz transformation; spin-gravity interaction

PACS numbers: $04.20 . \mathrm{Cv}, 04.20 .-\mathrm{q}, 04.20 . \mathrm{Fy}, 11.10 . \mathrm{Ef}, 11.10 .-\mathrm{z}$

*Electronic address: yqgu@fudan.edu.cn 


\section{Contents}

I. Introduction

II. Simplification of the Spinor Connection

III. Relations between Tetrad and Metric

IV. The Classical Approximation of Dirac Equation

V. Energy-Momentum Tensor of Spinors

VI. Discussion and Conclusion

Acknowledgments

References

\section{INTRODUCTION}

Dirac equation for spinor is a magic equation, which includes many secrets of Nature. The interaction between spinors and gravity is the most complicated and subtle interaction in the universe, which involves the basic problem to unified quantum theory and general relativity. The spinor connection has been constructed and researched in many literatures[1-6]. The spinor field is used to explain the accelerating expansion of the universe and dark matter and dark energy[7-12]. In the previous works, we usually used spinor covariant derivative directly, in which the spinor connection takes a compact form and its physical meaning becomes ambiguous. In this paper, by means of Clifford algebra, we split the spinor connection into geometrical and dynamical parts $\left(\Upsilon_{\mu}, \Omega_{\mu}\right)$ respectively. This form of connection is determined only by metric but independent of Dirac matrices. Only in this representation, we can clearly define classical concepts such as coordinate, speed, momentum and spin for a spinor, and then derive the classical mechanics in detail. $\Upsilon_{\mu} \in \Lambda^{1}$ only corresponds to the geometrical calculations, but $\Omega_{\mu} \in \Lambda^{3}$ leads to dynamical effects. $\Omega_{\mu}$ couples with the spin $S^{\mu}$ of a spinor, which provides location and navigation functions for a spinor with little energy. This term is also related with the origin of magnetic field of a celestial body[13]. So this form of connection is much helpful to understand the subtle relation between spinor and space-time. 
The classical theory for a spinor moving in gravitational field is firstly studied by Mathisson[14], and then developed by Papapetrou[15] and Dixon[16]. A detailed derivation can be found in [17]. By the commutator of the covariant derivative of the spinor $\left[\nabla_{\alpha}, \nabla_{\beta}\right]$, we get an extra approximate acceleration of the spinor as follows

$$
a_{\alpha}\left(x^{\mu}\right)=-\frac{\hbar}{4 m} R_{\alpha \beta \gamma \delta}\left(x^{\mu}\right) u^{\beta}\left(x^{\mu}\right) S^{\gamma \delta}\left(x^{\mu}\right)
$$

where $R_{\alpha \beta \gamma \delta}$ is the Riemann curvature, $u^{\alpha}$ 4-vector speed and $S^{\gamma \delta}$ the half commutator of the Dirac matrices.

(1.1) leads to the violation of Einstein's equivalence principle. This problem was discussed by many authors[17-24]. In [18], the exact Cini-Touschek transformation and the ultra-relativistic limit of the fermion theory were derived, but the Foldy-Wouthuysen transformation is not uniquely defined. The following calculations also show that, the usual covariant derivative $\nabla_{\mu}$ includes cross terms, which is not parallel to the speed $u^{\mu}$ of the spinor.

To study the coupling effect of spinor and space-time, we need the energy-momentum tensor(EMT) of spinor in curved space-time. The interaction of spinor and gravity is considered by H. Weyl as early as in 1929[25]. There are some approaches to the general expression of EMT of spinors in curved space-time[4, 9, 26, 27]. But the formalisms are usually quite complicated for practical calculation and different from each other. In [7-10, 12], the space-time is usually Friedmann-Lemaitre-Robertson-Walker type with diagonal metric. The energy-momentum tensor $T_{\mu \nu}$ of spinors can be directly derived from Lagrangian of the spinor field in this case. In [4, 26], according to the Pauli's theorem

$$
\delta \gamma^{\alpha}=\frac{1}{2} \gamma_{\beta} \delta g^{\alpha \beta}+\left[\gamma^{\alpha}, M\right]
$$

where $M$ is a traceless matrix related to the frame transformation, the EMT for Dirac spinor $\phi$ was derived as follows,

$$
T^{\mu \nu}=\frac{1}{2} \Re\left\langle\phi^{\dagger}\left(\gamma^{\mu} i \nabla^{\nu}+\gamma^{\nu} i \nabla^{\mu}\right) \phi\right\rangle,
$$

where $\phi^{\dagger}=\phi^{+} \gamma$ is the Dirac conjugation, $\nabla^{\mu}$ is the usual covariant derivatives for spinor. A detailed calculation for variation of action was performed in [9], and the results were a little different from (1.2) and (1.3).

The following calculation shows that, $M$ is still related with $\delta g^{\mu \nu}$, and provides nonzero contribution to $T^{\mu \nu}$ in general cases. The exact form of EMT is much more complex than (1.3), which includes some important effects overlooked previously. The covariant derivatives operator 
$i \nabla_{\mu}$ for spinor includes components in grade-3 Clifford algebra $\Lambda^{3}$ which is not parallel to the classical momentum $p_{\mu} \in \Lambda^{1}$. The derivation of rigorous $T_{\mu \nu}$ is quite difficult due to non-uniqueness representation and complicated formalism of vierbein or tetrad frames. In this paper, we give a systematical and detailed calculation for EMT of spinors. We clearly establish the relations between tetrad and metric at first, and then solve the Euler derivatives with respect to $g_{\mu \nu}$ to get explicit and rigorous form of $T_{\mu \nu}$.

From the results we find some new and interesting conclusions. Besides the usual kinetic energy momentum term, we find three kinds of other additional terms in EMT of bispinor. One is the self interactive potential, which acts like negative pressure. The other reflects the interaction of momentum $p^{\mu}$ with tetrad, which vanishes in classical approximation. The third is the spin-gravity coupling term $\Omega_{\alpha} S^{\alpha}$, which is a higher order infinitesimal in weak field, but becomes important in a neutron star. All these results are based on Clifford algebra decomposition of usual spin connection $\Gamma_{\mu}$ into geometrical part $\Upsilon_{\mu}$ and dynamical part $\Omega_{\mu}$, which not only makes calculation simpler, but also highlights their different physical meanings. In the calculation of tetrad formalism we find a new spinor coefficient table $S_{a b}^{\mu \nu}$, which plays an important role in the interaction of spinor with gravity and appears in many places.

This paper is an improvement and synthesis of the previous works arXiv:gr-qc/0610001 and arXiv:gr-qc/0612106, which were repeatedly rejected by the professional journals due to nonacademic reasons in physical society. The materials in this paper are organized as follows: In the next section, we specify notations and conventions used in the paper, and derive the spinor connections in form of Clifford algebra. In the third section, we set up the relations between tetrad and metric, which is the technical foundations of classical approximation of Dirac equation and EMT of spinor. We derive the classical approximation of spinor theory in section IV, and then calculate the EMT in section V. We give some simple discussions in the last section.

\section{SIMPLIFICATION OF THE SPINOR CONNECTION}

Clifford algebra is a unified language and efficient tool for physics. The variables and equations expressed by Clifford algebra have a neat and elegant form, and the calculation has a standard but simple procedure. At first we introduce some notations and conventions used in this paper. We take $\hbar=c=1$ as units. The element of space-time is described by

$$
d \mathbf{x}=\gamma_{\mu} d x^{\mu}=\gamma^{\mu} d x_{\mu}=\gamma_{a} \delta X^{a}=\gamma^{a} \delta X_{a}
$$


in which $\gamma_{a}$ stands for tetrad, and $\gamma^{a}$ for co-frame, which satisfy the following $C \ell_{1,3}$ Clifford algebra,

$$
\begin{gathered}
\gamma_{a} \gamma_{b}+\gamma_{b} \gamma_{a}=2 \eta_{a b}, \quad \gamma_{\mu} \gamma_{\nu}+\gamma_{\nu} \gamma_{\mu}=2 g_{\mu \nu}, \\
\gamma_{\mu}=f_{\mu}{ }^{a} \gamma_{a}, \quad \gamma^{\mu}=f_{a}^{\mu} \gamma^{a}, \quad \eta_{a b}=\operatorname{diag}(1,-1,-1,-1) .
\end{gathered}
$$

The relation between the local frame coefficient $\left(f_{a}^{\mu}, f_{\mu}{ }^{a}\right)$ and metric is given by

$$
f_{\mu}^{a} f_{b}^{\mu}=\delta_{b}^{a}, \quad f_{\mu}^{a} f_{a}^{\nu}=\delta_{\mu}^{\nu}, \quad f_{a}^{\mu} f_{b}^{\nu} \eta^{a b}=g^{\mu \nu}, \quad f_{\mu}^{a} f_{\nu}{ }^{b} \eta_{a b}=g_{\mu \nu}
$$

We use the Latin characters $(a, b \in\{0,1,2,3\})$ for the Minkowski indices, Greek characters $(\mu, \nu \in$ $\{0,1,2,3\})$ for the curvilinear indices, and $(j, k, l, m, n \in\{1,2,3\})$ for spatial indices. The Pauli and Dirac matrices in Minkowski space-time are given by

$$
\begin{aligned}
& \sigma^{a} \equiv\left\{\left(\begin{array}{ll}
1 & 0 \\
0 & 1
\end{array}\right),\left(\begin{array}{ll}
0 & 1 \\
1 & 0
\end{array}\right),\left(\begin{array}{cc}
0 & -i \\
i & 0
\end{array}\right),\left(\begin{array}{cc}
1 & 0 \\
0 & -1
\end{array}\right)\right\}, \\
& \tilde{\sigma}^{a} \equiv\left(\sigma^{0},-\vec{\sigma}\right), \quad \vec{\sigma}=\left(\sigma^{1}, \sigma^{2}, \sigma^{3}\right), \\
& \gamma^{a} \equiv\left(\begin{array}{cc}
0 & \tilde{\sigma}^{a} \\
\sigma^{a} & 0
\end{array}\right), \quad \gamma^{5}=\left(\begin{array}{cc}
I & 0 \\
0 & -I
\end{array}\right) \text {. }
\end{aligned}
$$

Since the Clifford algebra is isomorphic to the matrix algebra, we need not distinguish tetrad $\gamma^{a}$ and matrix $\gamma^{a}$ in calculation.

There are several definitions for Clifford algebra[28, 29]. Clifford algebra is also called geometric algebra. If the definition is directly related to geometric concepts, it will bring great convenience to the study and research of geometry[30, 31].

Definition 1 Assume the element of an $n=p+q$ dimensional space-time $\mathbb{M}^{p, q}$ over $\mathbb{R}$ is given by (2.1). The space-time is endowed with distance $d s=|d \mathbf{x}|$ and oriented volumes $d V_{k}$ calculated by

$$
\begin{aligned}
& d \mathbf{x}^{2}=\frac{1}{2}\left(\gamma_{\mu} \gamma_{\nu}+\gamma_{\nu} \gamma_{\mu}\right) d x^{\mu} d x^{\nu}=g_{\mu \nu} d x^{\mu} d x^{\nu}=\eta_{a b} \delta X^{a} \delta X^{b} \\
& d V_{k}=d \mathbf{x}_{1} \wedge d \mathbf{x}_{2} \wedge \cdots \wedge d \mathbf{x}_{k}=\gamma_{\mu \nu \cdots \omega} d x_{1}^{\mu} d x_{2}^{\nu} \cdots d x_{k}^{\omega}, \quad(1 \leq k \leq n)
\end{aligned}
$$

in which the Minkowski metric $\left(\eta_{a b}\right)=\operatorname{diag}\left(I_{p},-I_{q}\right)$, and Grassmann basis $\gamma_{\mu \nu \cdots \omega}=\gamma_{\mu} \wedge \gamma_{\nu} \wedge \cdots \wedge$ $\gamma_{\omega} \in \Lambda^{k} \mathbb{M}^{p, q}$. Then the following number with basis

$$
C=c_{0} I+c_{\mu} \gamma^{\mu}+c_{\mu \nu} \gamma^{\mu \nu}+\cdots+c_{12 \cdots n} \gamma^{12 \cdots n}, \quad\left(\forall c_{k} \in \mathbb{R}\right)
$$

together with multiplication rule of basis given in (2.8) and associativity define the $2^{n}$-dimensional real universal Clifford algebra $C \ell_{p, q}$.

By straightforward calculation we have[6, 30, 31] 
Theorem 1 For $C \ell_{1,3}$, we have the following useful relations

$$
\begin{gathered}
I, \quad \gamma^{a}, \quad \gamma^{a b}=\frac{i}{2} \epsilon^{a b c d} \gamma_{c d} \gamma^{5}, \quad \gamma^{a b c}=i \epsilon^{a b c d} \gamma_{d} \gamma^{5}, \quad \gamma^{0123}=-i \gamma^{5} \\
\gamma^{\mu} \gamma^{\nu}=g^{\mu \nu}+\gamma^{\mu \nu}, \quad \gamma^{\mu \nu} \gamma^{\omega}=\gamma^{\mu} g^{\nu \omega}-\gamma^{\nu} g^{\mu \omega}+\gamma^{\mu \nu \omega}
\end{gathered}
$$

For Dirac equation in curved space-time without torsion, we have[1-5, 32],

$$
\gamma^{\mu}\left(i \nabla_{\mu}-e A_{\mu}\right) \phi=m \phi, \quad \nabla_{\mu} \phi=\left(\partial_{\mu}+\Gamma_{\mu}\right) \phi
$$

in which the spinor connection is given by

$$
\Gamma_{\mu} \equiv \frac{1}{4} \gamma_{\nu} \gamma_{; \mu}^{\nu}=\frac{1}{4} \gamma^{\nu} \gamma_{\nu ; \mu}=\frac{1}{4} \gamma^{\nu}\left(\partial_{\mu} \gamma_{\nu}-\Gamma_{\mu \nu}^{\alpha} \gamma_{\alpha}\right)
$$

The total spinor connection $\gamma^{\mu} \Gamma_{\mu} \in \Lambda^{1} \cup \Lambda^{3}$.

Theorem 2 Dirac equation (2.13) can be rewritten in the following Hermitian form

$$
\left(\alpha^{\mu} \hat{p}_{\mu}-S^{\mu} \Omega_{\mu}\right) \phi=m \gamma^{0} \phi
$$

in which $\alpha^{\mu}$ is current operator, $\hat{p}_{\mu}$ momentum and $S^{\mu}$ spin operator,

$$
\alpha^{\mu}=\operatorname{diag}\left(\sigma^{\mu}, \tilde{\sigma}^{\mu}\right), \quad \hat{p}_{\mu}=i\left(\partial_{\mu}+\Upsilon_{\mu}\right)-e A_{\mu}, \quad S^{\mu}=\frac{1}{2} \operatorname{diag}\left(\sigma^{\mu},-\widetilde{\sigma}^{\mu}\right)
$$

where $\Upsilon_{\mu}$ is Keller connection and $\Omega_{\mu}$ Gu-Nester potential, they are respectively defined as

$$
\begin{aligned}
\Upsilon_{\nu} & \equiv \frac{1}{2} f_{a}^{\mu}\left(\partial_{\nu} f_{\mu}{ }^{a}-\partial_{\mu} f_{\nu}{ }^{a}\right)=\frac{1}{2}\left[\partial_{\nu}(\ln \sqrt{g})-f_{a}^{\mu} \partial_{\mu} f_{\nu}{ }^{a}\right], \\
\Omega^{\alpha} & \equiv \frac{1}{2} f_{d}^{\alpha} f_{a}^{\mu} f_{b}^{\nu} \partial_{\mu} f_{\nu}{ }^{e} \epsilon^{a b c d} \eta_{c e}=\frac{1}{4 \sqrt{g}} \epsilon^{\alpha \mu \nu \omega} \eta_{a b} f_{\omega}{ }^{a}\left(\partial_{\mu} f_{\nu}{ }^{b}-\partial_{\nu} f_{\mu}{ }^{b}\right)
\end{aligned}
$$

Proof. By (2.11) and (2.12), we have following Clifford calculus

$$
\begin{aligned}
\gamma^{\mu} \Gamma_{\mu} & =\frac{1}{4} \gamma^{\mu} \gamma^{\nu}\left(\partial_{\mu} \gamma_{\nu}-\Gamma_{\mu \nu}^{\alpha} \gamma_{\alpha}\right)=\frac{1}{4}\left(g^{\mu \nu}+\gamma^{\mu \nu}\right)\left(\partial_{\mu} \gamma_{\nu}-\Gamma_{\mu \nu}^{\alpha} \gamma_{\alpha}\right) \\
& =\frac{1}{4}\left(\gamma_{; \mu}^{\mu}+\gamma^{\mu \nu} \partial_{\mu} \gamma_{\nu}\right)=\frac{1}{4}\left(\partial_{\mu} \gamma^{\mu}+\partial_{\mu} \ln (\sqrt{g}) \gamma^{\mu}\right)+\frac{1}{4} f^{\mu}{ }_{a} f^{\nu}{ }_{b} \partial_{\mu} f_{\nu}{ }^{c} \gamma^{a b} \gamma_{c} \\
& =\frac{1}{4}\left[\gamma^{a} \partial_{\mu} f_{a}^{\mu}+\left(f^{\nu}{ }_{a} \partial_{\mu} f_{\nu}{ }^{a}\right) \gamma^{\mu}\right]+\frac{1}{4} f^{\mu}{ }_{a} f^{\nu}{ }_{b} \partial_{\mu} f_{\nu}{ }^{d} \gamma^{a b} \gamma^{c} \eta_{c d} \\
& =\frac{1}{4} f_{a}^{\mu} \gamma^{\nu}\left(-\partial_{\mu} f_{\nu}{ }^{a}+\partial_{\nu} f_{\mu}{ }^{a}\right)+\frac{1}{4} f_{a}^{\mu} f_{b}^{\nu} \partial_{\mu} f_{\nu}{ }^{d}\left(\eta^{b c} \gamma^{a}-\eta^{a c} \gamma^{b}+\gamma^{a b c}\right) \eta_{c d} \\
& =\frac{1}{2} f^{\mu}{ }_{a} \gamma^{\nu}\left(\partial_{\nu} f_{\mu}{ }^{a}-\partial_{\mu} f_{\nu}{ }^{a}\right)+\frac{1}{4} f_{a}^{\mu} f_{b}^{\nu} \partial_{\mu} f_{\nu}{ }^{e} \gamma^{a b c} \eta_{c e} \\
& =\Upsilon_{\mu} \gamma^{\mu}+\frac{i}{2} \Omega^{\alpha} \gamma_{\alpha} \gamma^{5} .
\end{aligned}
$$

Substituting it into (2.13) and multiplying the equation by $\gamma^{0}$, we prove the theorem. 
The following discussion shows that, $\Upsilon_{\mu}$ and $\Omega_{\mu}$ have different physical meanings. $\partial_{\mu}+\Upsilon_{\mu}$ as a whole operator is similar to the covariant derivatives $\nabla_{\mu}$ for vector, it only has geometrical effect. But $\Omega_{\mu}$ couples with the spin of a particle and leads to the magnetic field of celestial body[13]. $\Omega_{\mu} \equiv 0$ is a necessary condition for the metric can be diagonalized. If the gravitational field is generated by a rotating ball, the corresponding metric, like the Kerr one, cannot be diagonalized. In this case, the spin-gravity coupling term has non-zero coupling effect. In axisymmetric and asymptotically flat space-time we have the line element in quasi-spherical coordinate system[33]

$$
\begin{gathered}
d \mathbf{x}=\gamma_{0} \sqrt{U}(d t+W d \varphi)+\sqrt{V}\left(\gamma_{1} d r+\gamma_{2} r d \theta\right)+\gamma_{3} \sqrt{U^{-1}} r \sin \theta d \varphi \\
d \mathbf{x}^{2}=U(d t+W d \varphi)^{2}-V\left(d r^{2}+r^{2} d \theta^{2}\right)-U^{-1} r^{2} \sin ^{2} \theta d \varphi^{2}
\end{gathered}
$$

in which $(U, V, W)$ is just functions of $(r, \theta)$. As $r \rightarrow \infty$ we have

$$
U \rightarrow 1-\frac{2 m}{r}, \quad W \rightarrow \frac{4 L}{r} \sin ^{2} \theta, \quad V \rightarrow 1+\frac{2 m}{r}
$$

where $(m, L)$ are mass and angular momentum of the star respectively. For common stars and planets we always have $r \gg m \gg L$. For example, we have $m \doteq 3 \mathrm{~km}$ for the sun. The nonzero tetrad coefficients of metric (2.20) are given by

$$
\left\{\begin{array}{l}
f_{t}^{0}=\sqrt{U}, f_{r}{ }^{1}=\sqrt{V}, f_{\theta}{ }^{2}=r \sqrt{V}, f_{\varphi}{ }^{3}=\frac{r \sin \theta}{\sqrt{U}}, f_{\varphi}{ }^{0}=\sqrt{U} W \\
f_{0}^{t}=\frac{1}{\sqrt{U}}, f_{1}^{r}=\frac{1}{\sqrt{V}}, f_{2}^{\theta}=\frac{1}{r \sqrt{V}}, f_{3}^{\varphi}=\frac{\sqrt{U}}{r \sin \theta}, f_{3}^{t}=\frac{-\sqrt{U} W}{r \sin \theta}
\end{array}\right.
$$

Substituting it into (2.19) we get

$$
\begin{aligned}
\Omega^{\alpha} & =f_{0}^{t} f_{1}^{r} f_{2}^{\theta} f_{3}^{\varphi}\left(0, \partial_{\theta} g_{t \varphi},-\partial_{r} g_{t \varphi}, 0\right) \\
& =\left(V r^{2} \sin \theta\right)^{-1}\left(0, \partial_{\theta}(U W),-\partial_{r}(U W), 0\right) \\
& \rightarrow \frac{4 L}{r^{4}}(0,2 r \cos \theta, \sin \theta, 0)
\end{aligned}
$$

By (2.24) we find that, the intensity of $\Omega^{\alpha}$ is proportional to the angular momentum of the star, and its force line is given by

$$
\frac{d x^{\mu}}{d s}=\Omega^{\mu} \Rightarrow \frac{d r}{d \theta}=\frac{2 r \cos \theta}{\sin \theta} \Leftrightarrow r=R \sin ^{2} \theta .
$$

(2.25) shows that, the force lines of $\Omega^{\alpha}$ is just the magnetic lines of a magnetic dipole. According to the above results, we know that the spin-gravity coupling potential of charged particles will certainly induce a macroscopic dipolar magnetic field for a star, and it should be approximately in accordance with the Schuster-Wilson-Blackett relation[13]. 
For diagonal metric, the metric is given by

$$
g_{\mu \nu}=\operatorname{diag}\left(N_{0}^{2},-N_{1}^{2},-N_{2}^{2},-N_{3}^{2}\right), \quad \sqrt{g}=N_{0} N_{1} N_{2} N_{3},
$$

where $N_{\mu}=N_{\mu}\left(x^{\alpha}\right)$. In this case we have $\Omega_{\mu} \equiv 0$ and

$$
\gamma^{\mu}=\left(\frac{\gamma^{0}}{N_{0}}, \frac{\gamma^{1}}{N_{1}}, \frac{\gamma^{2}}{N_{2}}, \frac{\gamma^{3}}{N_{3}}\right), \quad \Upsilon_{\mu}=\frac{1}{2} \partial_{\mu} \ln \left(\frac{\sqrt{g}}{N_{\mu}}\right)
$$

For Dirac equation in Schwarzschild metric,

$$
g_{\mu \nu}=\operatorname{diag}\left(B(r),-A(r),-r^{2},-r^{2} \sin ^{2} \theta\right)
$$

we have

$$
\gamma^{\mu}=\left(\frac{\gamma^{0}}{\sqrt{B}}, \frac{\gamma^{1}}{\sqrt{A}}, \frac{\gamma^{2}}{r}, \frac{\gamma^{3}}{r \sin \theta}\right), \quad \Upsilon_{\mu}=\left(1, \frac{1}{r}+\frac{B^{\prime}}{4 B}, \frac{1}{2} \cot \theta, 0\right) .
$$

The Dirac equation for free spinor is given by

$$
i\left(\frac{\gamma^{0}}{\sqrt{B}} \partial_{t}+\frac{\gamma^{1}}{\sqrt{A}}\left(\partial_{r}+\frac{1}{r}+\frac{B^{\prime}}{4 B}\right)+\frac{\gamma^{2}}{r}\left(\partial_{\theta}+\frac{1}{2} \cot \theta\right)+\frac{\gamma^{3}}{r \sin \theta} \partial_{\varphi}\right) \phi=m \phi
$$

Set $A=B=1$, we get Dirac equation in spherical coordinate system. In contrast with the spinor in the Cartesian coordinate system, the spinor in the (2.30) includes an implicit rotational transformation.

\section{RELATIONS BETWEEN TETRAD AND METRIC}

Different from the cases of vector and tensor, in general relativity the equation of spinor fields depends on the local tetrad frame. The tetrad $\gamma^{\alpha}$ can be only determined by metric to an arbitrary Lorentz transformation. This situation makes the derivation of EMT quite complicated. In this section, we give an explicit representation of tetrad and derive the EMT of spinor based on this representation. For convenience to check the results by computer, we denote the element by $d x^{\mu}=(d x, d y, d z, c d t)$ and $\delta X^{a}=(\delta X, \delta Y, \delta Z, c \delta T)$.

For metric $g_{\mu \nu}$, not losing generality we assume that, in the neighborhood of $x^{\mu}, d x^{0}$ is time-like and $\left(d x^{1}, d x^{2}, d x^{3}\right)$ are space-like. This means $g_{00} \geq 0, g_{k k} \leq 0(k \neq 0)$, and the following definitions of $J_{k}$ are real numbers

$$
\begin{gathered}
J_{1}=\sqrt{-g_{11}}, J_{2}=\sqrt{\left|\begin{array}{ll}
g_{11} & g_{12} \\
g_{21} & g_{22}
\end{array}\right|}, J_{3}=\sqrt{-\left|\begin{array}{lll}
g_{11} & g_{12} & g_{13} \\
g_{21} & g_{22} & g_{23} \\
g_{31} & g_{32} & g_{33}
\end{array}\right|}, J_{0}=\sqrt{-\operatorname{det}(g)} . \\
u_{1}=\left|\begin{array}{ll}
g_{11} & g_{12} \\
g_{31} & g_{32}
\end{array}\right|, \quad u_{2}=\left|\begin{array}{ll}
g_{11} & g_{12} \\
g_{01} & g_{02}
\end{array}\right|, \quad u_{3}=\left|\begin{array}{ll}
g_{21} & g_{22} \\
g_{31} & g_{32}
\end{array}\right|
\end{gathered}
$$




$$
v_{1}=\left|\begin{array}{lll}
g_{12} & g_{13} & g_{10} \\
g_{22} & g_{23} & g_{20} \\
g_{32} & g_{33} & g_{30}
\end{array}\right|, \quad v_{2}=\left|\begin{array}{lll}
g_{11} & g_{13} & g_{10} \\
g_{21} & g_{23} & g_{20} \\
g_{31} & g_{33} & g_{30}
\end{array}\right|, \quad v_{3}=\left|\begin{array}{lll}
g_{11} & g_{12} & g_{10} \\
g_{21} & g_{22} & g_{20} \\
g_{31} & g_{32} & g_{30}
\end{array}\right| \text {. }
$$

The following conclusions can be checked by computer program.

Theorem 3 For LU decomposition of matrix $\left(g_{\mu \nu}\right)$

$$
\left(g_{\mu \nu}\right)=L\left(\eta_{a b}\right) L^{+}, \quad\left(g^{\mu \nu}\right)=U\left(\eta_{a b}\right) U^{+}, \quad U=L^{*}=\left(L^{+}\right)^{-1},
$$

with positive diagonal elements, we have the following unique solution

$$
\begin{gathered}
L=\left(L_{\mu}^{a}\right)=\left(\begin{array}{cccc}
-\frac{g_{11}}{J_{1}} & 0 & 0 & 0 \\
-\frac{g_{21}}{J_{1}} & \frac{J_{2}}{J_{1}} & 0 & 0 \\
-\frac{g_{31}}{J_{1}} & \frac{u_{1}}{J_{1} J_{2}} & \frac{J_{3}}{J_{2}} & 0 \\
-\frac{g_{01}}{J_{1}} & \frac{u_{2}}{J_{1} J_{2}} & -\frac{v_{3}}{J_{2} J_{3}} & \frac{J_{0}}{J_{3}}
\end{array}\right), \\
U=\left(U_{a}^{\mu}\right)=\left(\begin{array}{cccc}
\frac{1}{J_{1}} & \frac{g_{21}}{J_{2} J_{2}} & \frac{u_{3}}{J_{2} J_{3}} & \frac{v_{1}}{J_{3} J_{0}} \\
0 & \frac{J_{1}}{J_{2}} & -\frac{u_{1}}{J_{2} J_{3}} & -\frac{v_{2}}{J_{3} J_{0}} \\
0 & 0 & \frac{J_{2}}{J_{3}} & \frac{v_{3}}{J_{3} J_{0}} \\
0 & 0 & 0 & \frac{J_{3}}{J_{0}}
\end{array}\right) .
\end{gathered}
$$

Theorem 4 For any solution of tetrad (2.4) in matrix form $\left(f_{\mu}{ }^{a}\right)$ and $\left(f_{a}^{\mu}\right)$, there exists a local Lorentz transformation $\delta X^{\prime a}=\Lambda_{b}^{a} \delta X^{b}$ independent of $g_{\mu \nu}$, such that

$$
\left(f_{\mu}^{a}\right)=L \Lambda^{+}, \quad\left(f_{a}^{\mu}\right)=U \Lambda^{-1}
$$

where $\Lambda=\left(\Lambda_{b}^{a}\right)$ stands for the matrix of Lorentz transformation.

Proof. For any solution (2.4) we have

$$
\left(g_{\mu \nu}\right)=L\left(\eta_{a b}\right) L^{+}=\left(f_{\mu}^{a}\right)\left(\eta_{a b}\right)\left(f_{\mu}^{a}\right)^{+} \Leftrightarrow L^{-1}\left(f_{\mu}^{a}\right)\left(\eta_{a b}\right)\left(L^{-1}\left(f_{\mu}^{a}\right)\right)^{+}=\left(\eta_{a b}\right) .
$$

So we have a Lorentz transformation matrix $\Lambda=\left(\Lambda_{b}^{a}\right)$, such that

$$
L^{-1}\left(f_{\mu}^{a}\right)=\Lambda^{+} \Leftrightarrow\left(f_{\mu}^{a}\right)=L \Lambda^{+}, \text {or } f_{\mu}^{a}=L_{\mu}^{b} \Lambda_{b}^{a} .
$$

Similarly we have $\left(f_{a}^{\mu}\right)=U \Lambda^{-1}$. The proof is finished. 
The decomposition (3.4) is the Gram-Schmidt orthogonalization for vectors $d x^{\mu}$ in the order $d t \rightarrow d z \rightarrow d y \rightarrow d x$. In matrix form, by (3.4) we have $\delta X=L^{+} d x$ and

$$
\begin{aligned}
d s^{2}= & g_{\mu \nu} d x^{\mu} d x^{\nu}=\eta_{a b} \delta X^{a} \delta X^{b} \\
= & \left(L_{t}^{T} d t\right)^{2}-\left(L_{x}^{X} d x+L_{y}^{X} d y+L_{z}{ }^{X} d z+L_{t}{ }^{X} d t\right)^{2} \\
& -\left(L_{y}^{Y} d y+L_{z}{ }^{Y} d z+L_{t}{ }^{Y} d t\right)^{2}-\left(L_{z}{ }^{Z} d z+L_{t}{ }^{Z} d t\right)^{2}
\end{aligned}
$$

(3.10) is a direct result of (3.5), but (3.10) manifestly shows the geometrical meanings of the tetrad components $L_{\mu}^{a}$. Obviously, (3.10) is also the method of completing the square to calculate the tetrad coefficients $f_{\mu}{ }^{a}$.

For $L U$ decomposition (3.6), we define a spinor coefficient table by

$$
S_{a b}^{\mu \nu} \equiv\left(\begin{array}{cccc}
0 & -U_{1}^{\{\mu} U_{2}^{\nu\}} & -U_{1}^{\{\mu} U_{3}^{\nu\}} & -U_{1}^{\{\mu} U_{0}^{\nu\}} \\
U_{2}^{\{\mu} U_{1}^{\nu\}} & 0 & -U_{2}^{\{\mu} U_{3}^{\nu\}} & -U_{2}^{\{\mu} U_{0}^{\nu\}} \\
U_{3}^{\{\mu} U_{1}^{\nu\}} & U_{3}^{\{\mu} U_{2}^{\nu\}} & 0 & -U_{3}^{\{\mu} U_{0}^{\nu\}} \\
U_{0}^{\{\mu} U_{1}^{\nu\}} & U_{0}^{\{\mu} U_{2}^{\nu\}} & U_{0}^{\{\mu} U_{3}^{\nu\}} & 0
\end{array}\right)=-S_{b a}^{\mu \nu},
$$

in which

$$
U_{a}^{\{\mu} U_{b}^{\nu\}}=\frac{1}{2}\left(U_{a}^{\mu} U_{b}^{\nu}+U_{b}^{\nu} U_{a}^{\mu}\right)=U_{b}^{\{\mu} U_{a}^{\nu\}}
$$

$S_{a b}^{\mu \nu}$ is not a tensor for indices $(a, b)$, it is symmetrical for Riemann indices $(\mu, \nu)$ but antisymmetrical for Minkowski indices $(a, b)$. By representation of (3.5), (3.6) and relation (3.7), we can check the following results by straightforward calculation.

Theorem 5 For tetrad (3.7), we have

$$
\begin{aligned}
& \frac{\partial f_{\alpha}{ }^{n}}{\partial g_{\mu \nu}}=\frac{1}{4}\left(\delta_{\alpha}^{\mu} f_{m}^{\nu}+\delta_{\alpha}^{\nu} f_{m}^{\mu}\right) \eta^{n m}+\frac{1}{2} S_{a b}^{\mu \nu} f_{\alpha}{ }^{a} \eta^{n b} . \\
& \frac{\partial f_{a}^{\alpha}}{\partial g_{\mu \nu}}=-\frac{1}{4}\left(f_{a}^{\mu} g^{\alpha \nu}+f_{a}^{\nu} g^{\mu \alpha}\right)-\frac{1}{2} S_{a b}^{\mu \nu} f_{n}^{\alpha} \eta^{n b} .
\end{aligned}
$$

Or equivalently,

$$
\begin{aligned}
\frac{\partial \gamma_{\alpha}}{\partial g_{\mu \nu}} & =\frac{1}{4}\left(\delta_{\alpha}^{\mu} \gamma^{\nu}+\delta_{\alpha}^{\nu} \gamma^{\mu}\right)+\frac{1}{2} S_{a b}^{\mu \nu} f_{\alpha}{ }^{a} \gamma^{b} \\
\frac{\partial \gamma^{\alpha}}{\partial g_{\mu \nu}} & =-\frac{1}{4}\left(g^{\mu \alpha} \gamma^{\nu}+g^{\nu \alpha} \gamma^{\mu}\right)-\frac{1}{2} S_{a b}^{\mu \nu} f_{n}^{\alpha} \gamma^{a} \eta^{n b}
\end{aligned}
$$

Or equivalently,

$$
\begin{aligned}
& \delta \gamma_{\alpha}=\frac{1}{2} \gamma^{\beta}\left(\delta g_{\alpha \beta}+S_{a b}^{\mu \nu} f_{\alpha}^{a} f_{\beta}^{b} \delta g_{\mu \nu}\right) \\
& \delta \gamma^{\lambda}=-\frac{1}{2} g^{\lambda \beta} \gamma^{\alpha}\left(\delta g_{\alpha \beta}+S_{a b}^{\mu \nu} f_{\alpha}^{a} f_{\beta}^{b} \delta g_{\mu \nu}\right)=-g^{\lambda \alpha} \delta \gamma_{\alpha}
\end{aligned}
$$


For any given vector $A^{\mu}$, we have

$$
\begin{aligned}
& A^{\alpha} \frac{\partial \gamma_{\alpha}}{\partial g_{\mu \nu}}=\frac{1}{4}\left(A^{\mu} \gamma^{\nu}+A^{\nu} \gamma^{\mu}\right)+\frac{1}{4} S_{a b}^{\mu \nu}\left(A^{a} \gamma^{b}-A^{b} \gamma^{a}\right) \\
& A_{\alpha} \frac{\partial \gamma^{\alpha}}{\partial g_{\mu \nu}}=-\frac{1}{4}\left(A^{\mu} \gamma^{\nu}+A^{\nu} \gamma^{\mu}\right)+\frac{1}{4} S_{a b}^{\mu \nu}\left(A^{a} \gamma^{b}-A^{b} \gamma^{a}\right)
\end{aligned}
$$

In $(3.13)-(3.20)$, we set $\frac{\partial \gamma^{\alpha}}{\partial g_{\mu \nu}}=\frac{\partial \gamma^{\alpha}}{\partial g_{\nu \mu}}=\frac{1}{2} \frac{d \gamma^{\alpha}}{d g_{\mu \nu}}$ for $\mu \neq \nu$ to get the tensor form. $\frac{d}{d g_{\mu \nu}}$ is the total derivative for $g_{\mu \nu}$ and $g_{\nu \mu}$. $S_{a b}^{\mu \nu}$ is transformed from (3.11).

It should be stressed again, $S_{a b}^{\mu \nu}$ is not a tensor for indices $(a, b)$, the following derivation only use the property $S_{a b}^{\mu \nu}=-S_{b a}^{\mu \nu}$. For concrete calculations, we should use (3.11). For $\Omega^{\alpha}$, we have

$$
\Omega^{d}=\frac{1}{4} \epsilon^{a b c d} f_{a}^{\alpha} S_{b c}^{\mu \nu} \partial_{\alpha} g_{\mu \nu}, \quad \Omega^{\alpha}=-\frac{1}{4} \epsilon^{d a b c} f_{d}^{\alpha} f^{\beta}{ }_{a} S_{b c}^{\mu \nu} \partial_{\beta} g_{\mu \nu} .
$$

\section{THE CLASSICAL APPROXIMATION OF DIRAC EQUATION}

In this section, we derive the classical mechanics for a charged spinor moving in gravity, and disclose the physical meaning of connections $\Upsilon_{\mu}$ and $\Omega_{\mu}$. In the Hamiltonian system of quantum mechanics, we need the coordinate system of global realistic simultaneity, that is, the Gu's natural coordinate system(NCS)[34]

$$
d s^{2}=g_{t t} d t^{2}-\bar{g}_{k l} d x^{k} d x^{l}, \quad d \tau=\sqrt{g_{t t}} d t=f_{t}^{0} d t, \quad d V=\sqrt{\bar{g}} d^{3} x
$$

In which $d s$ is the proper time element, $d \tau$ the Newton's absolute cosmic time element and $d V$ the absolute volume element of the space at time $t$. NCS generally exists and the global simultaneity is unique. Only in NCS we can clearly establish the Hamiltonian formalism and calculate the Nöther charges. In NCS, we have

$$
f_{t}^{0}=\sqrt{g_{t t}}, \quad f_{0}^{t}=\frac{1}{\sqrt{g_{t t}}}, \quad \gamma_{t}=\sqrt{g_{t t}} \gamma_{0}, \quad \gamma^{t}=\frac{1}{\sqrt{g_{t t}}} \gamma^{0}
$$

Then by (2.17) we get

$$
\Upsilon_{\mu}=\frac{1}{2}\left(\partial_{t} \ln \sqrt{\bar{g}}, f_{k}{ }^{a} \partial_{j} f^{j}{ }_{a}+\partial_{k} \ln \sqrt{g}\right), \quad \Upsilon^{t}=g^{t t} \Upsilon_{t}, \quad \Upsilon^{k}=-\bar{g}^{k l} \Upsilon_{l}
$$

In NCS, to lift and lower the index of a vector means $\Omega^{t}=g^{t t} \Omega_{t}, \Omega^{k}=-\bar{g}^{k l} \Omega_{l}$.

More generally, we consider Dirac equation with electromagnetic potential $e A^{\mu}$ and nonlinear potential $N(\check{\gamma})=\frac{1}{2} w \check{\gamma}^{2}$, where $\check{\gamma}=\phi^{+} \gamma_{0} \phi$. Then $(2.15)$ can be rewritten in Hamiltonian formalism

$$
i \alpha^{t} \nabla_{t} \phi=\mathbf{H} \phi, \quad \mathbf{H}=-\alpha^{k} \hat{p}_{k}+e \alpha^{t} A_{t}+S^{\mu} \Omega_{\mu}+\left(m-N^{\prime}\right) \gamma_{0}
$$


where $\mathbf{H}$ is the Hamiltonian or energy of the spinor, $\alpha^{t}=f_{0}^{t} \alpha^{0}=\left(\sqrt{g_{t t}}\right)^{-1}$ and $\nabla_{\mu}=\partial_{\mu}+\Upsilon_{\mu}$.

In traditional quantum theory, we simultaneously take coordinate, speed, momentum and wave function of a particle as original concepts. This situation is the origin of logical confusion. As a matter of fact, only wave function $\phi$ is independent concept and dynamical equation (4.4) is fundamental in logic. Other concepts of the particle should be defined by $\phi$ and (4.4). Similarly to the case in flat space-time[35, 36], we define some classical concepts for the spinor.

Definition 2 the coordinate $\vec{X}$ and speed $\vec{v}$ of the spinor is defined as

$$
X^{k}(t) \equiv \int_{R^{3}} x^{k}|\phi|^{2} \sqrt{\bar{g}} d^{3} x=\int_{R^{3}} x^{k} q^{t} \sqrt{g} d^{3} x, \quad v^{k} \equiv \frac{d}{d \tau} X^{k}=f_{0}^{t} \frac{d}{d t} X^{k},
$$

where $R^{3}$ stands for the total simultaneous hypersurface, $q^{\mu}=\phi^{+} \alpha^{\mu} \phi=\check{\alpha}^{\mu}$ is the current.

By definition (4.5) and current conservation law $q_{; \mu}^{\mu}=(\sqrt{g})^{-1} \partial_{\mu}\left(q^{\mu} \sqrt{g}\right)=0$, we have

$$
\begin{aligned}
v^{j} & =f_{0}^{t} \int_{R^{3}} x^{j} \partial_{t}\left(q^{t} \sqrt{g}\right) d^{3} x=-f_{0}^{t} \int_{R^{3}} x^{j} \partial_{k}\left(q^{k} \sqrt{g}\right) d^{3} x \\
& =f_{0}^{t} \int_{R^{3}} q^{j} \sqrt{g} d^{3} x \rightarrow \int_{R^{3}} q^{j} \sqrt{\bar{g}} d^{3} x .
\end{aligned}
$$

Since a spinor has only a very tiny structure, together with normalizing condition $\int_{R^{3}} q^{t} \sqrt{g} d^{3} x=1$, we get the classical point-particle model for the spinor as[36]

$$
q^{\mu} \rightarrow u^{\mu} \sqrt{1-v^{2}} \delta^{3}(\vec{x}-\vec{X}), \quad v^{2}=\bar{g}_{k l} v^{k} v^{l}, \quad u^{\mu}=\frac{d X^{\mu}}{d s}=\frac{v^{\mu}}{\sqrt{1-v^{2}}},
$$

where the Dirac- $\delta$ means $\int_{R^{3}} \delta^{3}(\vec{x}-\vec{X}) \sqrt{\bar{g}} d^{3} x=1$.

Theorem 6 For any Hermitian operator $\hat{P}, P \equiv \int_{R^{3}} \sqrt{\bar{g}} \phi^{+} \hat{P} \phi d^{3} x$ is real for any $\phi$. We have the following generalized Ehrenfest theorem,

$$
\frac{d P}{d t}=\Re \int_{R^{3}} \sqrt{g} \phi^{+}\left(\alpha^{t} \partial_{t} \hat{P}-i f_{0}^{t}\left[\hat{P}, f_{t}^{0}\right] \boldsymbol{H}+i[\boldsymbol{H}, \hat{P}]\right) \phi d^{3} x,
$$

where $\Re$ means taking the real part.

Proof. By (4.3) and (4.4), we have

$$
\begin{aligned}
\frac{d P}{d t}= & \frac{d}{d t} \int_{R^{3}} \sqrt{\bar{g}} \phi^{+} \hat{P} \phi d^{3} x \\
= & \Re \int_{R^{3}} \sqrt{\bar{g}}\left(\phi^{+}\left(\partial_{t} \hat{P}\right) \phi+i\left(i \partial_{t} \phi\right)^{+} \hat{P} \phi-i \phi^{+} \hat{P}\left(i \partial_{t} \phi\right)+\phi^{+} \hat{P} \phi \partial_{t} \ln \sqrt{\bar{g}}\right) d^{3} x \\
= & \Re \int_{R^{3}} \sqrt{\bar{g}}\left(\phi^{+}\left(\partial_{t} \hat{P}\right) \phi+i f_{t}^{0}(\mathbf{H} \phi)^{+} \hat{P} \phi-i \phi^{+} \hat{P}\left(f_{t}{ }^{0} \mathbf{H} \phi\right)\right) d^{3} x \\
= & \Re \int_{R^{3}} \sqrt{g} \phi^{+}\left(\alpha^{t} \partial_{t} \hat{P}-i f_{0}^{t}\left[\hat{P}, f_{t}^{0}\right] \mathbf{H}+i[\mathbf{H}, \hat{P}]\right) \phi d^{3} x \\
& +\Re \int_{R^{3}} \sqrt{g} \phi^{+}\left(\partial_{k} \alpha^{k}+\alpha^{k} \partial_{k} \ln \sqrt{g}-2 \alpha^{k} \Upsilon_{k}\right) \hat{P} \phi d^{3} x \\
= & \Re \int_{R^{3}} \sqrt{g} \phi^{+}\left(\alpha^{t} \partial_{t} \hat{P}-i f_{0}^{t}\left[\hat{P}, f_{t}^{0}\right] \mathbf{H}+i[\mathbf{H}, \hat{P}]\right) \phi d^{3} x .
\end{aligned}
$$


Then we prove (4.8). The proof clearly shows the connection $\Upsilon^{\mu}$ has only geometrical effect, which cancels the derivatives of $\sqrt{g}$. Obviously, we cannot get (4.8) from conventional definition of spinor connection $\Gamma_{\mu}$.

Definition 3 The 4-dimensional momentum of the spinor is defined by

$$
p^{\mu}=\Re \int_{R^{3}}\left(\phi^{+} \hat{p}^{\mu} \phi\right) \sqrt{\bar{g}} d^{3} x
$$

For a spinor at energy eigenstate, we have classical approximation $p^{\mu}=m u^{\mu}$, where $m$ defines the classical inertial mass of the spinor.

Theorem 7 For momentum of the spinor $p_{\mu}=\Re \int_{R^{3}} \sqrt{\bar{g}} \phi^{+} \hat{p}_{\mu} \phi d^{3} x$, we have

$$
\frac{d}{d \tau} p_{\mu}=f_{0}^{t} \Re \int_{R^{3}} \sqrt{g}\left(e F_{\mu \nu} q^{\nu}+\check{S}^{a} \partial_{\mu} \Omega_{a}-\partial_{\mu} N-\phi^{+}\left(\partial_{\mu} \alpha^{\nu}\right) \hat{p}_{\nu} \phi\right) d^{3} x
$$

in which

$$
F_{\mu \nu}=\partial_{\mu} A_{\nu}-\partial_{\nu} A_{\mu}, \quad \check{S}^{a}=\phi^{+} S^{a} \phi
$$

Proof. Substituting $\hat{P}=\hat{p}_{\mu}$ and $\mathbf{H} \phi=\alpha^{t} i \nabla_{t} \phi$ into (4.8), by straightforward calculation we get

$$
\begin{aligned}
\frac{d}{d \tau} p_{\mu}= & f_{0}^{t} \Re \int_{R^{3}} \sqrt{g} \phi^{+}\left(-e \alpha^{t} \partial_{t} A_{\mu}-\left(\partial_{\mu} \alpha^{t}\right) i \nabla_{t}+\alpha^{k} \partial_{k} \hat{p}_{\mu}\right) \phi d^{3} x \\
& +f_{0}^{t} \Re \int_{R^{3}} \sqrt{g} \phi^{+}\left(\partial_{\mu}\left(-\alpha^{k} \hat{p}_{k}+e \alpha^{t} A_{t}+S^{\nu} \Omega_{\nu}-N^{\prime} \gamma_{0}\right)\right) \phi d^{3} x \\
= & f_{0}^{t} \Re \int_{R^{3}} \sqrt{g}\left(e F_{\mu \nu} q^{\nu}+\phi^{+} \partial_{\mu}\left(S^{\nu} \Omega_{\nu}\right) \phi-\partial_{\mu} N\right) d^{3} x-K_{\mu},
\end{aligned}
$$

in which

$$
K_{\mu}=f_{0}^{t} \Re \int_{R^{3}} \sqrt{g} \phi^{+}\left(\partial_{\mu} \alpha^{\nu}\right) \hat{p}_{\nu} \phi d^{3} x
$$

By $S^{\mu} \Omega_{\mu}=S^{a} \Omega_{a}$, we prove the theorem.

For a spinor at particle state[36], by classical approximation $q^{\mu} \rightarrow v^{\mu} \delta^{3}(\vec{x}-\vec{X})$ and local Lorentz transformation, we have respectively

$$
\begin{aligned}
\int_{R^{3}} e F_{\mu \nu} q^{\nu} \sqrt{g} d^{3} x & \rightarrow f_{t}^{0} e F_{\mu \nu} u^{\nu} \sqrt{1-v^{2}} \\
\int_{R^{3}} \phi^{+} S^{a} \phi\left(\partial_{\mu} \Omega_{a}\right) \sqrt{g} d^{3} x & \rightarrow f_{t}^{0} \bar{S}^{a} \partial_{\mu} \Omega_{a} \sqrt{1-v^{2}}=f_{t}^{0} \partial_{\mu}\left(\bar{S}^{a} \Omega_{a}\right) \sqrt{1-v^{2}} \\
\int_{R^{3}} \partial_{\mu} N \sqrt{g} d^{3} x & =\int_{R^{3}} \partial_{\mu}(N \sqrt{g}) d^{3} x-\int_{R^{3}} N \Gamma_{\mu \nu}^{\nu} \sqrt{g} d^{3} x \\
& \rightarrow \delta_{\mu}^{t} \frac{d}{d t}\left(f_{t}^{0} \bar{w} \sqrt{1-v^{2}}\right)-f_{t}^{0} \Gamma_{\mu \nu}^{\nu} \bar{w} \sqrt{1-v^{2}}
\end{aligned}
$$


in which the proper parameters $\bar{S}^{a}=\int_{R^{3}} \phi^{+} S^{a} \phi d^{3} X$ is almost a constant, $\bar{S}^{a}$ equals to $\pm \frac{1}{2} \hbar$ in one direction but vanishes in other directions. $\bar{w}=\int_{R^{3}} N d^{3} X$ is scale dependent. Then (4.11) becomes

$$
\frac{d}{d s} p_{\mu} \rightarrow e F_{\mu \nu} u^{\nu}+\partial_{\mu}\left(\bar{S}^{\nu} \Omega_{\nu}\right)+\bar{w}\left(\Gamma_{\mu \alpha}^{\alpha}-\delta_{\mu}^{t} \frac{d}{d t} \zeta\right)-\frac{K_{\mu}}{\sqrt{1-v^{2}}}
$$

where $\zeta=\ln \left(f_{t}^{0} \bar{w} \sqrt{1-v^{2}}\right)$.

Now we prove the following classical approximation of $K_{\mu}$,

$$
K_{\mu} \rightarrow-\frac{1}{2}\left(\partial_{\mu} g_{\alpha \beta}\right) m u^{\alpha} u^{\beta} \sqrt{1-v^{2}}
$$

For $L U$ decomposition of metric, by (3.14) we have

$$
\frac{\partial f_{a}^{\nu}}{\partial g_{\alpha \beta}}=-\frac{1}{4}\left(f_{a}^{\alpha} g^{\nu \beta}+f_{a}^{\beta} g^{\alpha \nu}\right)-\frac{1}{2} S_{a b}^{\alpha \beta} f_{n}^{\nu} \eta^{n b}
$$

where $S_{a b}^{\mu \nu}=-S_{b a}^{\mu \nu}$ is anti-symmetrical for indices $(a, b)$. Thus we have

$$
\begin{aligned}
\left(\partial_{\mu} \alpha^{\nu}\right) \hat{p}_{\nu} & =\partial_{\mu} g_{\alpha \beta} \frac{\partial f_{a}^{\nu}}{\partial g_{\alpha \beta}} \alpha^{a} \hat{p}_{\nu}=\partial_{\mu} g_{\alpha \beta}\left(-\frac{1}{4}\left(\alpha^{\alpha} \hat{p}^{\beta}+\alpha^{\beta} \hat{p}^{\alpha}\right)-\frac{1}{2} S_{a b}^{\alpha \beta} f_{n}^{\nu} \eta^{n b} \alpha^{a} \hat{p}_{\nu}\right) \\
& =-\frac{1}{4} \partial_{\mu} g_{\alpha \beta}\left(\left(\alpha^{\alpha} \hat{p}^{\beta}+\alpha^{\beta} \hat{p}^{\alpha}\right)+2 S_{a b}^{\alpha \beta} \alpha^{a} \hat{p}^{b}\right) .
\end{aligned}
$$

For classical approximation we have

$$
\check{\alpha}^{a}=\phi^{+} \alpha^{a} \phi \rightarrow v^{a} \delta^{3}(\vec{x}-\vec{X}), \quad \hat{p}^{b} \phi \rightarrow m u^{b} \phi, \quad S_{a b}^{\alpha \beta}=-S_{b a}^{\alpha \beta}
$$

Substituting (4.22) into (4.21), we get

$$
\int_{R^{3}} \sqrt{g} \phi^{+}\left(\partial_{\mu} \alpha^{\nu}\right) \hat{p}_{\nu} \phi d^{3} x \rightarrow-\frac{1}{2} f_{t}^{0}\left(\partial_{\mu} g_{\alpha \beta}\right) p^{\alpha} u^{\beta} \sqrt{1-v^{2}}
$$

So (4.19) holds.

In the central coordinate system of the spinor, by relations

$$
\Gamma_{\alpha \beta}^{\nu}=\frac{1}{2} g^{\mu \nu}\left(\partial_{\alpha} g_{\mu \beta}+\partial_{\beta} g_{\mu \alpha}-\partial_{\mu} g_{\alpha \beta}\right), \quad \frac{d}{d \tau} g_{\mu \nu}=\sqrt{1-v^{2}} u^{\alpha} \partial_{\alpha} g_{\mu \nu}
$$

it is easy to check

$$
g_{\mu \nu} \Gamma_{\alpha \beta}^{\nu} p^{\alpha} u^{\beta} \sqrt{1-v^{2}}-p^{\nu} \frac{d g_{\mu \nu}}{d \tau}=-\frac{1}{2}\left(\partial_{\mu} g_{\alpha \beta}\right) p^{\alpha} u^{\beta} \sqrt{1-v^{2}}
$$

Substituting (4.25) into (4.19) we get

$$
K_{\mu} \rightarrow g_{\mu \nu} \Gamma_{\alpha \beta}^{\nu} p^{\alpha} u^{\beta} \sqrt{1-v^{2}}-p^{\nu} \frac{d g_{\mu \nu}}{d \tau} .
$$

Substituting (4.26) and $d s=\sqrt{1-v^{2}} d \tau$ into (4.18), we get Newton's second law for the spinor

$$
\frac{d}{d s} p^{\mu}+\Gamma_{\alpha \beta}^{\mu} p^{\alpha} u^{\beta}=g^{\alpha \mu}\left(e F_{\alpha \beta} u^{\beta}+\bar{w}\left(\Gamma_{\alpha \beta}^{\beta}-\delta_{\alpha}^{t} \frac{d}{d t} \ln \zeta\right)+\partial_{\alpha}\left(\bar{S}^{\nu} \Omega_{\nu}\right)\right)
$$


The classical mass $m$ weakly depends on speed $v$ if $\bar{w} \neq 0$.

By the above derivation we find that, Newton's second law is not as simple as it looks like, because its universal validity depends on many subtle and compatible relations of the spinor equation. A complicated partial differential equation system (4.4) can be reduced to a 6-dimensional dynamics (4.5) and (4.27), the world is a miracle designed elaborately. If the spin-gravity coupling potential $S_{\mu} \Omega^{\mu}$ and nonlinear potential $\bar{w}$ can be ignored, (4.27) satisfies 'mass shell constraint' $\frac{d}{d t}\left(p^{\mu} p_{\mu}\right)=0[35-37]$. In this case, the classical mass of the spinor is a constant and the free spinor moves along geodesic. In some sense, only vector potential is strictly compatible with Newtonian mechanics and Einstein's principle of equivalence.

Clearly, the additional acceleration in (4.27) $\Omega_{\mu} \in \Lambda^{3}$ is different from that in (1.1), which is in $\Lambda^{2}$. The approximation to derive $(1.1) \hbar \rightarrow 0$ may be inadequate, because $\hbar$ is a universal constant acting as unit of physical variables. If $\bar{w}=0,(4.27)$ obviously holds in all coordinate system due to the covariant form, although we derive (4.27) in NCS. However, if $\bar{w}>0$ is large enough for dark spinor, its trajectories will manifestly deviate from geodesics, so the dark halo in a galaxy is automatically separated from ordinary matter. Besides, the nonlinear potential is scale dependent[38].

For many body problem, dynamics of the system should be juxtaposed (4.4) due to the superposition of Lagrangian,

$$
i \alpha^{t}\left(\partial_{t}+\Upsilon_{t}\right) \phi_{n}=\mathbf{H}_{n} \phi_{n}, \quad \mathbf{H}_{n}=-\alpha^{k} \hat{p}_{k}+e \alpha^{t} A_{t}+\left(m_{n}-N_{n}^{\prime}\right) \gamma_{0}+\Omega_{\mu} S^{\mu}
$$

The coordinate, speed and momentum of $n$-th spinor are defined by

$$
\vec{X}_{n}(t)=\int_{R^{3}} \vec{x} q_{n}^{t} \sqrt{g} d^{3} x, \quad \vec{v}_{n}=\frac{d}{d \tau} \vec{X}_{n}, \quad p_{n}^{\mu}=\Re \int_{R^{3}} \phi_{n}^{+} \hat{p}^{\mu} \phi_{n} \sqrt{\bar{g}} d^{3} x .
$$

The classical approximation condition for point-particle model reads,

$$
q_{n}^{\mu} \rightarrow u_{n}^{\mu} \sqrt{1-v_{n}^{2}} \delta^{3}\left(\vec{x}-\vec{X}_{n}\right), \quad u_{n}^{\mu} \equiv \frac{d X_{n}^{\mu}}{d s_{n}}=\left(1, \vec{v}_{n}\right) / \sqrt{1-v_{n}^{2}} .
$$

Repeating the derivation from (4.18) to (4.22), we get classical dynamics for each spinor,

$$
\frac{d}{d s_{n}} p_{n}^{\mu}+\Gamma_{\alpha \beta}^{\mu} p_{n}^{\alpha} u_{n}^{\beta}=g^{\alpha \mu}\left(e_{n} F_{\alpha \beta} u_{n}^{\beta}+\bar{w}_{n}\left(\Gamma_{\alpha \beta}^{\beta}-\delta_{\alpha}^{t} \frac{d}{d t} \ln \zeta_{n}\right)+\partial_{\alpha}\left(\bar{S}^{\nu} \Omega_{\nu}\right)\right) \text {. }
$$

\section{ENERGY-MOMENTUM TENSOR OF SPINORS}

Similarly to the case of metric $g_{\mu \nu}$, the definition of Ricci tensor can also differ by a negative sign. We take the definition as follows

$$
R_{\mu \nu} \equiv \partial_{\alpha} \Gamma_{\mu \nu}^{\alpha}-\partial_{\mu} \Gamma_{\nu \alpha}^{\alpha}-\Gamma_{\mu \beta}^{\alpha} \Gamma_{\nu \alpha}^{\beta}+\Gamma_{\mu \nu}^{\alpha} \Gamma_{\alpha \beta}^{\beta}, \quad R=g^{\mu \nu} R_{\mu \nu}
$$


For a spinor in gravity, the Lagrangian of the coupling system is given by

$$
\mathcal{L}=\frac{1}{2 \kappa}(R-2 \Lambda)+\mathcal{L}_{m}, \quad \mathcal{L}_{m}=\Re\left\langle\phi^{+} \alpha^{\mu} \hat{p}_{\mu} \phi\right\rangle-\phi^{+} \Omega_{\mu} S^{\mu} \phi-m \phi^{+} \gamma^{0} \phi+N
$$

in which $\kappa=8 \pi G, \Lambda$ is the cosmological constant, and $N=\frac{1}{2} w \check{\gamma}^{2}$ the nonlinear potential. Variation of the Lagrangian (5.2) with respect to $g_{\mu \nu}$, we get Einstein's field equation

$$
G^{\mu \nu}+\Lambda g^{\mu \nu}+\kappa T^{\mu \nu}=0, \quad G^{\mu \nu} \equiv R^{\mu \nu}-\frac{1}{2} g^{\mu \nu} R=-\frac{\delta(R \sqrt{g})}{\sqrt{g} \delta g_{\mu \nu}} .
$$

where $\frac{\delta}{\delta g_{\mu \nu}}$ is the Euler derivatives, and $T^{\mu \nu}$ is EMT of the spinor defined by

$$
T^{\mu \nu}=-2 \frac{\delta\left(\mathcal{L}_{m} \sqrt{g}\right)}{\sqrt{g} \delta g_{\mu \nu}}=-2 \frac{\partial \mathcal{L}_{m}}{\partial g_{\mu \nu}}+2\left(\partial_{\alpha}+\Gamma_{\alpha \gamma}^{\gamma}\right) \frac{\partial \mathcal{L}_{m}}{\partial\left(\partial_{\alpha} g_{\mu \nu}\right)}-g^{\mu \nu} \mathcal{L}_{m}
$$

By detailed calculation we have

Theorem 8 For the spinor $\phi$ with nonlinear potential $N(\check{\gamma})$, the total EMT is given by

$$
\begin{aligned}
T^{\mu \nu} & =\frac{1}{2} \Re\left\langle\phi^{+}\left(\alpha^{\mu} \hat{p}^{\nu}+\alpha^{\nu} \hat{p}^{\mu}+2 S_{a b}^{\mu \nu} \alpha^{a} \hat{p}^{b}\right) \phi\right\rangle+g^{\mu \nu}\left(N^{\prime} \check{\gamma}-N\right)+K^{\mu \nu}+\widetilde{K}^{\mu \nu}, \\
K^{\mu \nu} & =\frac{1}{2} \epsilon^{a b c d} \check{S}_{d}\left(\frac{1}{2} f_{a}^{\beta} S_{b c}^{\mu \nu} g^{\lambda \kappa}+\frac{\partial\left(f_{a}^{\beta} S_{b c}^{\mu \nu}\right)}{\partial g_{\lambda \kappa}}-\frac{\partial\left(f_{a}^{\beta} S_{b c}^{\lambda \kappa}\right)}{\partial g_{\mu \nu}}\right) \partial_{\beta} g_{\lambda \kappa}, \\
\widetilde{K}^{\mu \nu} & =\frac{1}{4} \epsilon^{a b c d} S_{c d}^{\mu \nu}\left(\partial_{a} \check{S}_{b}-\partial_{b} \check{S}_{a}\right), \quad \check{S}_{\mu} \equiv \phi^{+} S_{\mu} \phi .
\end{aligned}
$$

Proof. The Keller connection $i \Upsilon_{\alpha}$ is anti-Hermitian and actually vanishes in $\Re\left\langle\phi^{+} \alpha^{\alpha} \hat{p}_{\alpha} \phi\right\rangle$. By (5.4) and (3.20), we get the component of EMT related to the kinematic energy as

$$
\begin{aligned}
T_{p}^{\mu \nu} & \equiv-2 \frac{\delta}{\delta g_{\mu \nu}} \Re\left\langle\phi^{+} \alpha^{\alpha} \hat{p}_{\alpha} \phi\right\rangle=-2 \Re\left\langle\phi^{+}\left(\frac{\partial \alpha^{\alpha}}{\partial g_{\mu \nu}}\right)\left(i \partial_{\alpha}-e A_{\alpha}\right) \phi\right\rangle \\
& =\frac{1}{2} \Re\left\langle\phi^{+}\left(\alpha^{\mu} \hat{p}^{\nu}+\alpha^{\nu} \hat{p}^{\mu}+2 S_{a b}^{\mu \nu} \alpha^{a} \hat{p}^{b}\right) \phi\right\rangle,
\end{aligned}
$$

where we take $A_{\mu}$ as independent variable. By (3.21) we get the variation related with spin-gravity coupling potential as

$$
\begin{gathered}
\frac{\partial\left(\phi^{+} \Omega^{d} S_{d} \phi\right)}{\partial g_{\mu \nu}}=\frac{1}{4} \epsilon^{a b c d} \check{S}_{d} \frac{\partial\left(f_{a}^{\alpha} S_{b c}^{\lambda \kappa}\right)}{\partial g_{\mu \nu}} \partial_{\alpha} g_{\lambda \kappa}, \\
\left(\partial_{\alpha}+\Gamma_{\alpha \beta}^{\beta}\right) \frac{\partial\left(\phi^{+} \Omega^{d} S_{d} \phi\right)}{\partial\left(\partial_{\alpha} g_{\mu \nu}\right)}=\frac{1}{4} \epsilon^{a b c d}\left(\partial_{\alpha}+\Gamma_{\alpha \beta}^{\beta}\right)\left(f_{a}^{\alpha} S_{b c}^{\mu \nu} \check{S}_{d}\right) \\
=\frac{1}{4} \epsilon^{a b c d}\left[S_{b c}^{\mu \nu} \partial_{a} \check{S}_{d}+\check{S}_{d}\left(\frac{\partial\left(f_{a}^{\alpha} S_{b c}^{\mu \nu}\right)}{\partial g_{\lambda \kappa}}+\frac{1}{2} f_{a}^{\alpha} S_{b c}^{\mu \nu} g^{\lambda \kappa}\right) \partial_{\alpha} g_{\lambda \kappa}\right] .
\end{gathered}
$$

Then we have the EMT for term $\Omega^{\mu} \check{S}_{\mu}$ as

$$
T_{s}^{\mu \nu}=-2 \frac{\partial\left(\Omega^{d} \check{S}_{d}\right)}{\partial g_{\mu \nu}}+2\left(\partial_{\alpha}+\Gamma_{\alpha \beta}^{\beta}\right) \frac{\partial\left(\Omega^{d} \check{S}_{d}\right)}{\partial\left(\partial_{\alpha} g_{\mu \nu}\right)}=K^{\mu \nu}+\widetilde{K}^{\mu \nu}
$$


Substituting Dirac equation (2.15) into (5.2), we get $\mathcal{L}_{m}=N-N^{\prime} \check{\gamma}$. For nonlinear potential $N=\frac{1}{2} w \check{\gamma}^{2}$, we have $\mathcal{L}_{m}=-N$. Substituting all the results into (5.4), we prove the theorem.

For EMT of compound systems, we have the following useful theorem[39].

Theorem 9 Assume matter consists of two subsystems $I$ and $I I$, namely $\mathcal{L}_{m}=\mathcal{L}_{I}(\phi)+\mathcal{L}_{I I}(\psi)$, then we have

$$
T^{\mu \nu}=T_{I}^{\mu \nu}+T_{I I}^{\mu \nu}
$$

If the subsystems I and II have not interaction with each other, namely,

$$
\frac{\delta}{\delta \psi} \mathcal{L}_{I}(\phi)=\frac{\delta}{\delta \phi} \mathcal{L}_{I I}(\psi)=0
$$

then the two subsystems have independent energy-momentum conservation laws respectively,

$$
T_{I ; \nu}^{\mu \nu}=0, \quad T_{I I ; \nu}^{\mu \nu}=0 .
$$

For classical approximation of EMT, we have $\phi^{+} S_{a b}^{\mu \nu} \alpha^{a} \hat{p}^{b} \phi \rightarrow S_{a b}^{\mu \nu} u^{a} p^{b}=0$. By the symmetry of the spinor, the proper value $\int_{R^{3}} \widetilde{K}^{\mu \nu} d^{3} X=0$. By the structure and covariance, it seems that

$$
K^{\mu \nu}=k_{1} \check{S}_{\alpha} \Omega^{\alpha} g^{\mu \nu}+k_{2}\left(\Omega^{\mu} \check{S}^{\nu}+\Omega^{\nu} \check{S}^{\mu}\right)
$$

where $k_{1}, k_{2}$ are constants to be determined. Noticing that the energy of spin-gravity interaction is just $\check{S}^{\mu} \Omega_{\mu}$. Besides, if $A^{\mu}=0$, the spinor is an independent system and its energy-momentum conservation law $T_{; \nu}^{\mu \nu}=0$ holds, so its classical approximation should give $(4.27)$ as $F_{\mu \nu}=0$. This means we should have $k_{1}=1, k_{2}=0$ or $K_{t}^{t}=\check{S}^{\mu} \Omega_{\mu}$. How to strictly prove this result is still a problem. However, for the classical approximation of (5.5), by the summation of energy we certainly have the total EMT as

$$
T^{\mu \nu} \rightarrow\left[m u^{\mu} u^{\nu}+\left(\bar{S}^{\alpha} \Omega_{\alpha}+\bar{w}\right) g^{\mu \nu}\right] \sqrt{1-v^{2}} \delta^{3}(\vec{x}-\vec{X})
$$

$\bar{w}>0$ acts like negative pressure, which is scale dependent.

Some previous works usually use one spinor to represent matter field. This may be not the case, because spinor fields only has a very tiny structure. Only to represent one particle by one spinor field, the matter model can be comparable with general relativity, classical mechanics and quantum mechanics[12, 35, 40]. By the superposable property of Lagrangian, the many body system should be described by the following Lagrangian

$$
\mathcal{L}_{m}=\sum_{n}\left(\Re\left\langle\phi_{n}^{+} \alpha^{\mu} \hat{p}_{\mu} \phi_{n}\right\rangle-\check{S}_{n}^{\mu} \Omega_{\mu}-m_{n} \check{\gamma}_{n}+N_{n}\right)
$$


The classical approximation of EMT becomes

$$
T^{\mu \nu} \rightarrow \sum_{n}\left[m_{n} u_{n}^{\mu} u_{n}^{\nu}+\left(\bar{w}_{n}+\check{S}_{n}^{\alpha} \Omega_{\alpha}\right) g^{\mu \nu}\right] \sqrt{1-v_{n}^{2}} \delta^{3}\left(\vec{x}-\vec{X}_{n}\right),
$$

which leads to the EMT for average field of spinor fluid as follows

$$
T^{\mu \nu}=(\rho+P) U^{\mu} U^{\nu}+(W-P) g^{\mu \nu}
$$

The self potential becomes negative pressure $W$, which takes the place of cosmological constant $\Lambda$ in Einstein's field equation. $W$ has very important effects in astrophysics[39-41].

\section{DISCUSSION AND CONCLUSION}

From the calculation of this paper, we can find that Clifford algebra is indeed a unified language and efficient tool to describe the laws of Nature. To represent the physical and geometric quantities by Clifford algebra, the formalism is neat and elegant, and the calculation and derivation are simple and standard. The decomposition of spinor connection into $\Upsilon_{\mu}$ and $\Omega_{\mu}$ by Clifford algebra, not only makes the calculation simpler, but also highlights their different physical meanings. $\Upsilon_{\mu} \in \Lambda^{1}$ only corresponds to geometric calculations simlilar to Levi-Civita connection, but $\Omega_{\mu} \in \Lambda^{3}$ results in physical effects. $\Omega_{\mu}$ is coupled with the spin of spinor field, which provides position and navigation functions for the spinor, and is the origin of the celestial magnetic field. $\Omega_{\mu} \equiv 0$ is a necessary condition of the diagonalizablity of metric, which seems to be also sufficient.

In the theoretical analysis of the spinor equation and its classical approximation, we must use Gu's natural coordinate system with realistic cosmic time. This is a coordinate system with universal applicability and profound philosophical significance, which can clarify many misunderstandings about the concept of space-time. The energy-momentum tensor of the spinor field involves the specific representation of the tetrad. Through the $L U$ decomposition of metric, we set up the clear relationship between the frame and metric, and then derive the exact EMT of spinor. In the derivation, we discover a new non-tensor spinor coefficient table $S_{a b}^{\mu \nu}$, which has some wonderful properties and appears in many places in the spinor theory, but the specific physical significance needs to be further studied.

We usually use limits such as $\hbar \rightarrow 0$ and $c \rightarrow \infty$ in classical approximation of quantum mechanics. In some cases, such treatment is inappropriate. $(\hbar, c)$ are constant units for physical variables, how can they take limits. In the natural unit system used in this paper or the dimensionless equations, we even do not know where the constants are. We can only make approximations such 
as $|v| \ll c$ or (4.7) while the average radius of the spinor is much smaller than its moving scale. Most paradoxes and puzzles in physics are caused by such ambiguous statements or overlapping concepts in different logical systems. A detailed discussion of these issues is given in [36, 42].

This paper clearly shows how general relativity, quantum mechanics and classical mechanics are all compatible. Newton's second law is not as simple as it looks like, its universal validity depends on many subtle and compatible relations of the spinor equation as shown in section IV. A complicated Dirac equation of spinor can be reduced to a 6-dimensional ordinary differential dynamics is not a trivial event, which implies that the world is a miracle designed elaborately. In fact, all the fundamental physical theories can be unified in the following framework expressed by the Clifford algebra[36, 38]:

A1. The element of space-time is described by

$$
d \mathbf{x}=\gamma_{\mu} d x^{\mu}=\gamma_{a} \delta X^{a}
$$

in which $\gamma_{a}$ and $\gamma_{\mu}$ satisfy the $C \ell_{1,3}$ Clifford algebra (2.2).

A2. The dynamics for a definite physical system takes the form as

$$
\gamma^{\mu} \partial_{\mu} \Psi=\mathcal{F}(\Psi)
$$

in which $\Psi=\left(\psi_{1}, \psi_{2}, \cdots, \psi_{n}\right)^{T}$, and $\mathcal{F}(\Psi)$ consists of some Clifford numbers of $\Psi$, so that the total equation is covariant.

A3. Nature is consistent, i.e., for all solutions to (6.2) we always have $\forall \psi_{k} \in L^{\infty}$.

\section{Acknowledgments}

It is my pleasure to acknowledge Prof. James M. Nester for his enlightening discussions and encouragement. I once encountered the difficulty in derivation of energy-momentum tensor. He suggested me to learn Clifford algebra, which is the key to solve the problem.

[1] M. Sachs, General relativity and matter (Ch.3), D. Reidel, 1982.

[2] W. L. Bade, H. Jehle, Rev. Mod. Phys.25(3), (1953)714

[3] P. G. Bergmann, Phys. Rev. 107(2), (1957)624

[4] D. R. Brill, J. A. Wheeler, Rev. Mod. Phys. 29(3), (1957)465

[5] J. P. Crawford, Adv. Appl. Cliff. Alg. 2(1), (1992)75 
[6] Y. Q. Gu, Space-Time Geometry and Some Applications of Clifford Algebra in Physics. Adv. Appl. Clifford Algebras, 2018, 28(4):79.

[7] R. Rakhi, G.V. Vijayagovindan, K. Indulekha, A Cosmological Model with Fermionic Field, Int. J. Mod. Phys. A25:2735-2746, 2010, arXiv:0912.1222

[8] M. O. Ribas, F. P. Devecchi, G. M. Kremer, Fermions as sources of accelerated regimes in cosmology, Phys. Rev. D72 (2005) 123502, ArXiv:gr-qc/0511099.

[9] Ch. G. Boehmer, J. Burnett, D. F. Mota, D. J. Shaw, Dark spinor models in gravitation and cosmology, JHEP 1007:053, 2010, arXiv:1003.3858

[10] B. Saha, Nonlinear Spinor field in isotropic space-time and dark energy models, The European Phys. J Plus 131: 242 (2016)

[11] H. Wei Spinor dark energy and cosmological coincidence problem. Physics Letters B, 2011, 695(1-4):307311.

[12] Y. Q. Gu, A Cosmological Model with Dark Spinor Source, Int. J. Mod. Phys. A22:4667-4678(2007), gr-qc/0610147

[13] Y. Q. Gu, A New Explanation for the Origin of Magnetic Field of Celestial Bodies. Natural Science(in Chinese), 2019, 7(6):464-470. Preprints 2020, 2020020166 (doi: 10.20944/preprints202002.0166.v1)

[14] M. Mathisson, Acta Phys. Pol. 6, 163 (1937).

[15] A. Papapetrou, Proc. R. Soc. London 209, 248 (1951).

[16] W.G. Dixon, Phil. Trans. R. Soc. London A277, 59 (1974).

[17] P.M. Alsing, G.J. Stephenson Jr, and Patrick Kilian, Spin-induced non-geodesic motion, gyroscopic precession, Wigner rotation and EPR correlations of massive spin-1/2 particles in a gravitational field, arXiv:0902.1396

[18] Yuri N. Obukhov, On gravitational interaction of fermions, Fortsch. Phys. 50 (2002)711-716, arXiv:grqc/0112080

[19] H. Behera, P. C. Naik, Gravitomagnetic Moments and Dynamics of Dirac's (spin 1/2) fermions in flat space-time Maxwellian Gravity, Int. J. Mod. Phys. A19 (2004)4207-4230, arXiv:gr-qc/0304084.

[20] I. B. Khriplovich, A. A. Pomeransky, Gravitational Interaction of Spinning Bodies, Center-of-Mass Coordinate and Radiation of Compact Binary Systems, Phys.Lett. A216 (1996)7, arXiv:gr-qc/9602004.

[21] B. Mashhoon, D. Singh, Dynamics of extended spinning masses in a gravitational field, Phys. Rev. D74, 124006 (2006).

[22] B. Mashhoon, Neutron interferometry in a rotating frame of reference, Phys. Rev. Lett. 61, 2639-2642 (1988).

[23] F. W. Hehl, W. T. Ni, Inertial effects of a Dirac particle, Phys. Rev. D 42, 2045-2048 (1990).

[24] B. J. Venema, P. K. Majumder, S. K. Lamoreaux, B. R. Heckel and E. N. Fortson, Search for a coupling of the Earth's gravitational field to nuclear spins in atomic mercury, Phys. Rev. Lett. 68, 135-138 (1992).

[25] H. Weyl, Gravitation and the eletron, PNAS 15(4) 323-334(1929) 
[26] H. Arthur Weldon, Fermions without vierbeins in curved space-time, Phys. Rev. D63 (2001) 104010, arXiv:gr-qc/0009086

[27] H. B. Zhang, Note on the EMT for general mixed tensor-spinor fields, Commun. Theor. Phys. 44 (2005) 1007-1010

[28] P. Lounesto, Clifford Algebras and Spinors, Cambridge Univ. Press, Cambridge 2001.

[29] D. S. Shirokov, Clifford algebras and their applications to Lie groups and spinors, Proceedings of the Nineteenth International Conference on Geometry, Integrability and Quantization, Ivailo M. Mladenov and Akira Yoshioka, eds. (Sofia: Avangard Prima, 2018), 11-53, arXiv:1709.06608

[30] Y. Q. Gu, A Note on the Representation of Clifford Algebra, Preprints 2020, 2020020466 (doi: 10.20944/preprints202002.0466.v1).

[31] Y. Q. Gu, Some Applications of Clifford Algebra in Geometry, Preprints 2020, 2020020140 (doi:10.20944/preprints202002.0140.v1)

[32] J. M. Nester, Special orthonormal frames, J. Math. Phys. 33, 910 (1992)

[33] Y. Q. Gu, The Series Solution to the Metric of Stationary Vacuum with Axisymmetry. Chinese Physics B, 2008, 19(3):90-100.

[34] Y. Q. Gu, Natural Coordinate System in Curved Space-time, J. Geom. Symmetry Phys. 47 (2018) 51-62, arXiv:gr-qc/0612176, doi: 10.7546/jgsp-47-2018-51-62

[35] Y. Q. Gu, New Approach to N-body Relativistic Quantum Mechanics, Int. J. Mod. Phys. A22:20072020(2007), arXiv:hep-th/0610153

[36] Y. Q. Gu, Local Lorentz Transformation and Mass-Energy Relation of Spinor, Physics Essays Vol.31, 1-6(2018), DOI.10.13140/RG.2.2.13229.38882. arXiv:hep-th/0701030

[37] P. M. Alsing, J. C. Evans and K. K. Nandi, The phase of a quantum mechanical particle in curved space-time, Gen. Rel. Grav. 33, 1459-1487 (2001); gr-qc/0010065.

[38] Y. Q. Gu, Clifford Algebra, Lorentz Transformation and Unified Field Theory, Adv. Appl. Clifford Algebras 28(2):37, (2018)

[39] Y. Q. Gu, Energy-Momentum Tensor and Parameters in Cosmological Model, Preprints 2020, 2020020366 (doi:10.20944/preprints202002.0366.v1)

[40] Y. Q. Gu, Functions of State for Spinor Gas in General Relativity, OALib. J., 4, e3953 (2017), arXiv:0711.1243

[41] Y. Q. Gu, Nonlinear Spinors as the Candidate of Dark Matter, OALib. J. 4, e3954 (2017), arXiv:0806.4649

[42] Y. Q. Gu, Some Subtle Concepts in Fundamental Physics, Physics Essays Vol. 30: Pages 356-363(2017), arXiv:0901.0309 